\title{
Desenvolvimento cognitivo e de linguagem expressiva em um par de gêmeos dizigóticos: influência da síndrome de Down e da prematuridade associada ao muito baixo peso
}

\author{
Expressive language and cognitive development in a dizygotic twin pair: \\ influence of Down syndrome and prematurity combined with very \\ low birth-weight
}

\author{
Fabíola Custódio Flabiano ${ }^{1}$, Karina Elena Cadioli Bernardis Bühler ${ }^{2}$, Suelly Cecilia Olivan Limongi ${ }^{3}$
}

\begin{abstract}
RESUMO
O objetivo deste estudo foi descrever o desenvolvimento cognitivo e de linguagem expressiva de uma criança com síndrome de Down (SD) e seu gemelar, ambos nascidos pré-termo muito baixo peso (PTMBP), observando a influência da SD e da prematuridade associada ao muito baixo peso no processo de desenvolvimento durante o período sensório-motor. Participaram deste estudo um par de gêmeos dizigóticos, ambos do sexo masculino, nascidos PTMBP, sendo que apenas um apresentava SD. A idade inicial dos sujeitos foi de sete meses e quatro dias de idade cronológica e quatro meses e 21 dias de idade corrigida, considerando que nasceram com 29 semanas de gestação, pesando menos de 1500g. Os sujeitos foram acompanhados durante 12 meses em sessões quinzenais de 45 minutos e os dados foram registrados mensalmente em vídeo. O Protocolo para Observação do Desenvolvimento Cognitivo e de Linguagem Expressiva (PODCLE) foi utilizado para coleta e análise dos dados. Foram observadas diferenças significativas entre os gêmeos com relação ao desenvolvimento cognitivo e de linguagem expressiva. Apesar de apresentar melhor desempenho, o gemelar que não apresentava SD demonstrou um atraso importante, considerando-se as referências em relação ao desenvolvimento típico, evidenciando a influência da prematuridade associada ao muito baixo peso no desenvolvimento de linguagem e cognição. Os resultados obtidos para o outro gemelar sugerem que a SD levou ao aumento significativo deste atraso. A SD e a prematuridade associada ao muito baixo peso são condições que interferiram de forma negativa no desenvolvimento cognitivo e de linguagem expressiva apresentado pelo par de gêmeos estudado.
\end{abstract}

Descritores: Cognição; Linguagem; Gêmeos dizigóticos; Síndrome de Down; Prematuro; Recém-nascido de baixo peso; Relatos de casos

\section{INTRODUÇÃO}

Seguindo o modelo teórico proposto pela Epistemologia Genética, cuja base está apoiada nos princípios propostos

Trabalho realizado Laboratório de Investigação Fonoaudiológica em Síndromes e Alterações Sensório-Motoras da Faculdade de Medicina da Universidade de São Paulo - USP - São Paulo (SP), Brasil.

(1) Pesquisadora do Laboratório de Investigação Fonoaudiológica em Síndromes e Alterações Sensório-Motoras da Faculdade de Medicina da Universidade de São Paulo - USP - São Paulo (SP), Brasil; Pós-graduanda do Programa de Pós-Graduação em Ciências da Reabilitação da Faculdade de Medicina da Universidade de São Paulo - USP - São Paulo (SP), Brasil.

(2) Doutora, Fonoaudióloga do Hospital Universitário da Universidade de São Paulo USP - São Paulo (SP), Brasil.

(3) Livre-docente, Professora associada do Curso de Fonoaudiologia da Faculdade de Medicina da Universidade de São Paulo - USP - São Paulo (SP), Brasil.

Endereço para correspondência: Suelly Cecilia Olivan Limongi. R. Cipotânia, 51, Cidade Universitária, São Paulo - SP, CEP: 05360-160. E-mail: slimongi@usp.br

Recebido em: 18/6/2008; Aceito em: 14/12/2008 por Piaget $^{(1)}$, a ação leva à representação e esta, por sua vez, favorece a construção da linguagem. Assim, a linguagem está diretamente relacionada ao desenvolvimento cognitivo.

A criança apresenta, inicialmente durante o período sensório-motor, comportamentos de natureza reflexa. O exercício constante dessas ações reflexas, bem como sua coordenação e organização, irão levar à aquisição dos esquemas motores e estes, por sua vez, favorecerão a construção de esquemas mentais, contribuindo, assim, para a construção do conhecimento e da linguagem.

Nesse processo, a imitação desempenha papel fundamental, uma vez que revela a existência de condutas inteligentes, como o aprendizado da coordenação entre meios e fins. A imitação sensório-motora que permite à criança imitar na presença do modelo, evolui para uma imitação que requer a representação mental, denominada imitação diferida. A partir desse momento, a criança torna-se capaz de diferenciar significantes de significados e começa a utilizar símbolos e signos, expressando-os por meio de gestos, sinais e/ou palavras ${ }^{(2-4)}$. 
A criança com síndrome de Down (SD), devido a alterações orgânicas como a hipotonia, alterações endócrino-metabólicas, cardíacas e otorrinolaringológicas importantes, apresenta dificuldades na exploração do meio que influenciam diretamente suas experiências físicas, bem como a construção do conhecimento e da linguagem.

É relatado na literatura ${ }^{(5)}$ que as atividades dos bebês com SD que possibilitam a exploração do ambiente surgem com um atraso considerável, fator que dependerá muito da competência e desenvolvimento motor da criança. O brincar segue quase os mesmos padrões que os observados nas crianças normais, verificando-se algumas diferenças. A manipulação e exploração ocorrem com menor frequência, talvez pela menor habilidade motora.

Autores $^{(6)}$ relataram em seu estudo que as crianças com SD tendem a apresentar uma dificuldade característica em relação à linguagem oral, manifestando uma linguagem menos expressiva e produtiva quando comparadas a outras crianças com comprometimento intelectual decorrente de outras patologias.

Estudos $^{(7-8)}$ relatam que, devido às dificuldades quanto à linguagem expressiva e problemas específicos de articulação, tipicamente associados à síndrome, os gestos podem servir como um importante e efetivo meio de comunicação, apresentando-se por um longo período de tempo na criança com SD, quando comparada à criança com desenvolvimento normal.

Considerando que os primeiros meses de vida são de fundamental importância para a maturação neurológica, formação do vínculo afetivo e construção do conhecimento, crianças com histórico de prematuridade e muito baixo peso, apresentam alto risco para alterações do desenvolvimento cognitivo e, consequentemente, para o desenvolvimento de linguagem ${ }^{(9)}$.

Essas crianças são conhecidas por apresentarem risco para problemas cognitivos e comportamentais, incluindo QI mais baixos, dificuldades de aprendizagem, distração excessiva, hiperatividade, atrasos e desvios de linguagem além de déficits de funcionamento neuromotor. Além disso, essas crianças frequentemente apresentam alterações de fala e linguagem em idade escolar, mesmo na ausência de alterações neurológicas importantes $^{(10)}$.

Autores $^{(11)}$ demonstraram que os prematuros apresentam escores cognitivos inferiores aos de controles de termo, correlacionados ao peso de nascimento e à idade gestacional.

Estudo realizado em instituição nacional ${ }^{(12)}$ demonstrou diferenças estatisticamente significantes em relação à constituição da noção de permanência do objeto entre crianças pré-termo muito baixo peso (PTMBP) e crianças de termo, evidenciando o atraso (em média de dois a três meses de atraso) no desenvolvimento cognitivo apresentado pelas crianças nascidas PTMBP em relação ao grupo controle, durante o período sensório-motor.

Porém, são poucas as pesquisas que enfocam o desenvolvimento cognitivo e de linguagem em gêmeos, sendo inexistentes estudos que considerem a SD em co-ocorrência com a prematuridade e muito baixo peso.

O objetivo do presente estudo foi descrever o desenvolvimento cognitivo e de linguagem expressiva de uma criança com SD e seu gemelar, ambos nascidos PTMBP, observando a influência da SD e da prematuridade associada ao muito baixo peso no processo de desenvolvimento durante o período sensório-motor.

\section{APRESENTAÇÃO DO CASO CLÍNICO}

Esta pesquisa foi aprovada pela Comissão de Ética para Análise de Projetos de Pesquisa CAPPesq da Diretoria Clínica do Hospital das Clínicas e da Faculdade de Medicina da Universidade de São Paulo sob protocolo 397/05.

\section{Sujeitos}

Participaram deste estudo um par de gêmeos dizigóticos, ambos do sexo masculino, nascidos PTMBP, sendo que apenas um apresentava SD. A idade cronológica inicial dos sujeitos foi de sete meses e quatro dias e a final, 19 meses e 27 dias. Para fins de análise, foi considerada a idade corrigida (idade em que as crianças completariam 40 semanas de gestação), calculada com base na data da última menstruação da mãe (DUM). Assim, a idade corrigida inicial dos sujeitos foi de quatro meses e 21 dias e a final de 17 meses e 14 dias, considerando que nasceram com 29 semanas de gestação, pesando menos de $1500 \mathrm{~g}$.

Os sujeitos são participantes do Laboratório de Investigação Fonoaudiológica em Síndromes e Alterações SensórioMotoras (LIF-SASM) do Curso de Fonoaudiologia da FMUSP e recebem acompanhamentos clínico, pediátrico e otorrinolaringológico, sendo que o gemelar com SD realiza fisioterapia e terapia ocupacional. Segundo as avaliações clínicas, os sujeitos apresentam bom estado geral de saúde e não apresentam alterações auditivas e visuais.

\section{Material}

Para avaliação e acompanhamento dos sujeitos foi utilizado o Protocolo para Observação do Desenvolvimento Cognitivo e de Linguagem Expressiva (PODCLE) ${ }^{(13)}$.

Para registro das sessões terapêuticas, foi utilizado o protocolo de registros escritos, realizados ao final de cada sessão de terapia e transcrições das fitas de vídeo, gravadas em filmadora JVC.

Durante o período de intervenção fonoaudiológica, foram utilizados brinquedos variados, adequados à faixa etária e do desenvolvimento global dos sujeitos.

\section{Procedimentos}

Os sujeitos foram acompanhados durante 12 meses em sessões quinzenais de 45 minutos e os dados foram registrados mensalmente em vídeo e posteriormente transcritos e analisados segundo o PODCLE, contendo os seguintes itens: uso de esquemas isolados e coordenados; deslocamento dos objetos no espaço; permanência do objeto; imitação de esquemas motores; experiências com objetos novos; uso de objetos como meios; uso de esquemas simbólicos simples e combinados; produção de gestos dêiticos e representativos, acompanhados 
ou não por vocalizações; produção de verbalizações acompanhadas por gestos e produção de verbalizações isoladas.

As sessões eram realizadas sempre com a presença da mãe, para a qual foram dadas orientações e sugestões de brincadeiras adequadas à fase de desenvolvimento dos gêmeos, para serem realizadas em casa durante a quinzena.

A fundamentação teórica deste trabalho foi dada pela Epistemologia Genética, seguindo o modelo dialético-didático de observação ${ }^{(14)}$.

\section{DISCUSSÃO}

Por se tratar da descrição de um processo de desenvolvimento, os dados serão apresentados tanto de forma qualitativa, com a descrição das realizações e produções das crianças ao longo das sessões de observação, quanto de forma quantitativa, por meio da atribuição de pontos às realizações e produções observadas, conforme os procedimentos de análise dos dados propostos no PODCLE. Dessa forma, a trajetória de desenvolvimento percorrida por cada um dos sujeitos poderá ser evidenciada e também comparada às referências encontradas para crianças com desenvolvimento típico ${ }^{(15)}$.

Os resultados mostraram diferenças significativas entre os gêmeos, tanto em relação ao desenvolvimento cognitivo quanto em relação ao desenvolvimento de linguagem expressiva, sendo que o gemelar com SD apresentou pior desempenho que o gemelar que não apresenta SD (GNSD). Porém, ao considerarmos as referências encontradas para crianças com desenvolvimento típico, tanto o GSD quanto o GNSD apresentaram desempenho abaixo do esperado para a idade, durante todo o período de acompanhamento. Estes resultados podem ser melhor visualizados nas Figuras 1 e 2, os quais apresentam a evolução dos sujeitos mês a mês quanto ao desenvolvimento cognitivo e de linguagem expressiva, de acordo com a pontuação estabelecida no PODCLE.

$\mathrm{Na}$ avaliação inicial, de acordo com o PODCLE, o GSD encontrava-se na segunda fase do período sensório motor, enquanto o GNSD encontrava-se no início da terceira fase. Quanto à linguagem, os gêmeos apresentaram apenas vocalizações isoladas na avaliação inicial. Tais resultados podem ser melhor visualizados nos Quadros 1 e 2.

Durante o período de desenvolvimento compreendido entre a avaliação inicial e a avaliação final, a emergência dos indicadores do desenvolvimento cognitivo e de linguagem expressiva ocorreu nos sujeitos estudados da forma como mostram os Quadros 3 e 4.

$\mathrm{Na}$ avaliação final, o GSD encontrava-se na quinta fase de desenvolvimento sensório-motor, enquanto que seu irmão, já apresentava realizações cognitivas características da sexta fase, como o uso de esquemas simbólicos simples e a permanência do objeto, propriamente dita. Tais resultados podem ser melhor visualizados no Quadro 5.

Em relação à linguagem expressiva, na avaliação final, o GSD apresentou apenas uso por imitação simples de alguns gestos representativos e produção de vocalizações isoladas sem possibilidade de significado. O GNSD, por sua vez, apresentou imitação simples de gestos representativos mais complexos, imitação diferida de gestos dêiticos e representativos, bem como a produção de algumas verbalizações acompanhadas por gestos. Tais resultados podem ser melhor visualizados no Quadro 6.

Os resultados mostram que apesar de apresentar um melhor desempenho quanto ao desenvolvimento cognitivo

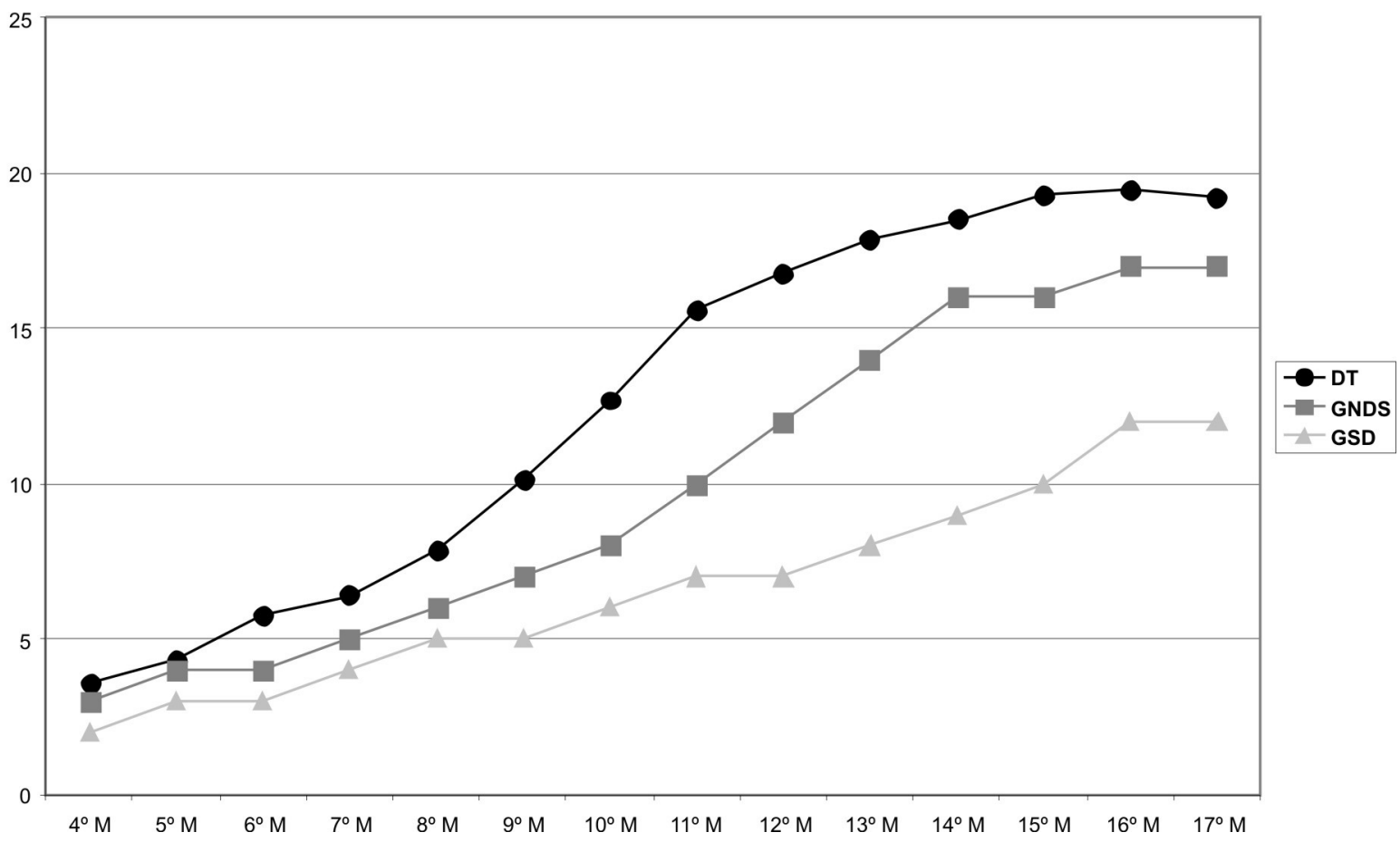

Legenda: DT= referência obtida para crianças com desenvolvimento típico; GNSD = gemelar que não apresenta síndrome de Down; GSD = gemelar que apresenta síndrome de Down

Figura 1. Desempenho dos gêmeos e as referências para o desenvolvimento típico quanto ao desenvolvimento cognitivo 


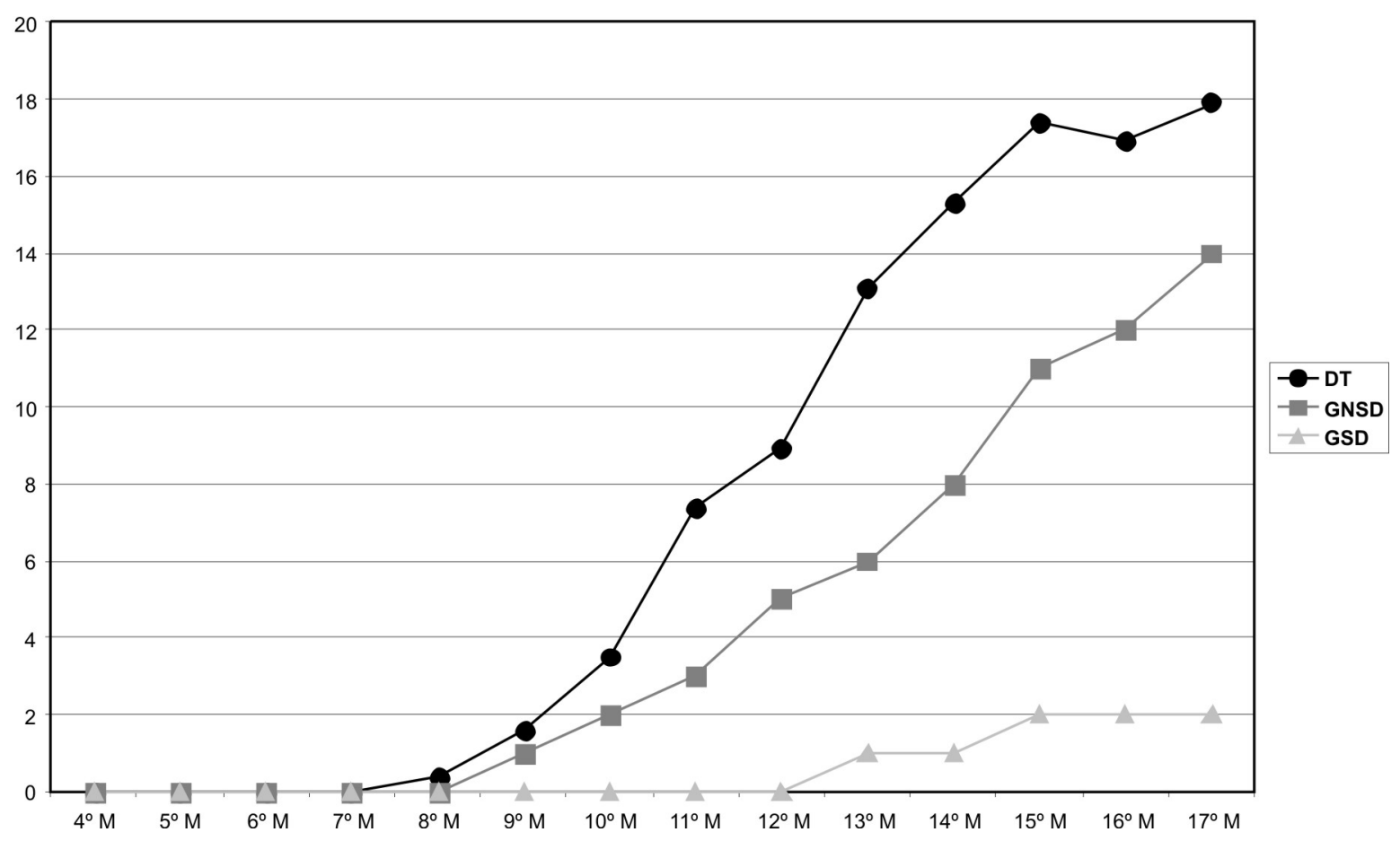

Legenda: DT= referência obtida para crianças com desenvolvimento típico; GNSD = gemelar que não apresenta síndrome de Down; GSD = gemelar que apresenta síndrome de Down

Figura 2. Desempenho dos gêmeos e as referências para o desenvolvimento típico quanto ao desenvolvimento de linguagem expressiva

Quadro 1. Comparação qualitativa entre os gêmeos em relação ao desenvolvimento cognitivo na avaliação inicial

\begin{tabular}{|c|c|c|c|}
\hline \multicolumn{4}{|c|}{ Avaliaçâo inicial - desenvolvimento cognitivo } \\
\hline \multicolumn{2}{|l|}{ GSD } & \multicolumn{2}{|l|}{ GNSD } \\
\hline Realizações da criança & $\begin{array}{c}\text { Fase de } \\
\text { desenvolvimento } \\
\text { cognitivo }\end{array}$ & Realizações da criança & $\begin{array}{c}\text { Fase de } \\
\text { desenvolvimento } \\
\text { cognitivo }\end{array}$ \\
\hline $\begin{array}{l}\text { - aplicação de esquemas isolados (olhar, } \\
\text { segurar) em objetos; } \\
\text { - acompanhamento do deslocamento do } \\
\text { objeto no espaço de forma incompleta }\end{array}$ & $\begin{array}{l}2^{\mathrm{a}} \text { fase do período } \\
\text { sensório-motor }\end{array}$ & $\begin{array}{l}\text { - aplicação de esquemas isolados e } \\
\text { coordenados (pegava o objeto e levava } \\
\text { à boca, olhava para o objeto enquanto o } \\
\text { chacoalhava. } \\
\text { - acompanhamento do deslocamento do } \\
\text { objeto no espaço de forma incompleta }\end{array}$ & $\begin{array}{c}3^{a} \text { fase do período } \\
\text { sensório-motor }\end{array}$ \\
\hline
\end{tabular}

Legenda: GSD = gemelar que apresenta síndrome de Down; GNSD = gemelar que não apresenta síndrome de Down

Quadro 2. Comparação qualitativa entre os gêmeos em relação ao desenvolvimento de linguagem expressiva na avaliação inicial

\begin{tabular}{|c|c|c|c|}
\hline \multicolumn{3}{|c|}{ Avaliaçâo inicial - desenvolvimento de linguagem expressiva } \\
\hline \multicolumn{1}{|c|}{ GSD } & \multicolumn{2}{c|}{ GNSD } \\
\hline \multicolumn{1}{|c|}{ Produções da criança } & $\begin{array}{l}\text { Conjunto de } \\
\text { produções }\end{array}$ & \multicolumn{1}{c|}{ Produções da criança } & produções de \\
\hline $\begin{array}{l}\text { - produção de vocalizações isoladas, sem } \\
\text { possibilidade de significação }\end{array}$ & Conjunto I & $\begin{array}{l}\text { - produção de vocalizações isoladas, sem } \\
\text { possibilidade de significação }\end{array}$ & Conjunto I \\
\hline
\end{tabular}

Legenda: GSD = gemelar que apresenta síndrome de Down; GNSD = gemelar que não apresenta síndrome de Down

e de linguagem expressiva em relação ao irmão, o GNSD apresentou-se atrasado, quando consideramos as referências em relação ao desenvolvimento típico, mesmo corrigindo sua idade, o que demonstra a influência da prematuridade associada ao muito baixo peso, para o desenvolvimento de linguagem e cognição.

Tal resultado corrobora outros estudos que apontam para atrasos no desenvolvimento cognitivo e de linguagem em crianças nascidas PTMBP ${ }^{(10-12)}$.

Como se pôde observar, a SD levou ao aumento signifi- 
Quadro 3. Comparação entre os gêmeos quanto à emergência dos indicadores de desenvolvimento cognitivo (de acordo com o PODCLE)

\begin{tabular}{|c|c|c|}
\hline \multicolumn{3}{|c|}{ Emergência dos indicadores de desenvolvimento cognitivo } \\
\hline \multirow[t]{2}{*}{ Indicadores } & \multicolumn{2}{|c|}{ Idade (em meses) } \\
\hline & GSD & GNSD \\
\hline Aplicação de esquemas isolados e coordenados & 5 meses & 4 meses \\
\hline Deslocamento dos objetos no espaço de forma completa & 7 meses & 5 meses \\
\hline Permanência do objeto, sem considerar os deslocamentos & 10 meses & 8 meses \\
\hline Permanência do objeto, considerando os deslocamentos visíveis & 15 meses & 11 meses \\
\hline Permanência do objeto, considerando os deslocamentos invisíveis & Mais de 17 meses & 15 meses \\
\hline Imitação de esquemas motores visíveis no próprio corpo & 14 meses & 10 meses \\
\hline Imitação de esquemas motores não visíveis no próprio corpo & Mais de 17 meses & 15 meses \\
\hline Imitação de esquemas motores que designam função aos objetos & Mais de 17 meses & 14 meses \\
\hline Conduta do suporte & 11 meses & 9 meses \\
\hline Conduta do barbante & 13 meses & 10 meses \\
\hline Conduta da vara & Mais de 17 meses & Mais de 17 meses \\
\hline Experiências com objetos novos & 12 meses & 16 meses \\
\hline Esquemas simbólicos simples aplicados no próprio corpo & Mais de 17 meses & 14 meses \\
\hline Esquemas simbólicos simples aplicados em objetos figurativos & Mais de 17 meses & 16 meses \\
\hline Esquemas simbólicos simples aplicados em objetos não figurativos & Mais de 17 meses & Mais de 17 meses \\
\hline Esquemas simbólicos combinados com duas ações & Mais de 17 meses & Mais de 17 meses \\
\hline Esquemas simbólicos combinados com 3 ou mais ações não ordenadas & Mais de 17 meses & Mais de 17 meses \\
\hline Esquemas simbólicos combinados com 3 ou mais ações ordenadas & Mais de 17 meses & Mais de 17 meses \\
\hline
\end{tabular}

Legenda: GSD = gemelar que apresenta síndrome de Down; GNSD = gemelar que não apresenta síndrome de Down

Quadro 4. Comparação entre os gêmeos quanto à emergência dos indicadores de desenvolvimento de linguagem expressiva (de acordo com o PODCLE)

\begin{tabular}{|c|c|c|}
\hline \multicolumn{3}{|c|}{ Emergência dos indicadores de desenvolvimento de linguagem expressiva } \\
\hline \multirow[t]{2}{*}{ Indicadores } & \multicolumn{2}{|c|}{ Idade (em meses) } \\
\hline & GSD & GNSD \\
\hline Uso de gestos dêiticos por imitação simples & 15 meses & 9 meses \\
\hline Uso de gestos dêiticos por imitação simples com vocalizações & Mais de 17 meses & 11 meses \\
\hline Uso de gestos dêiticos por imitação diferida & Mais de 17 meses & 12 meses \\
\hline Uso de gestos dêiticos por imitação diferida com vocalizações & Mais de 17 meses & 14 meses \\
\hline Uso de gestos representativos por imitação simples & 13 meses & 10 meses \\
\hline Uso de gestos representativos por imitação simples com vocalizações & Mais de 17 meses & 12 meses \\
\hline Uso de gestos representativos por imitação diferida & Mais de 17 meses & 13 meses \\
\hline Uso de gestos representativos por imitação diferida com vocalizações & Mais de 17 meses & 14 meses \\
\hline $\begin{array}{l}\text { Uso de gestos representativos acompanhados por sílabas com significado ou onomatopéias } \\
\text { vocais }\end{array}$ & Mais de 17 meses & 15 meses \\
\hline Produção de sílabas com significado acompanhadas por gestos & Mais de 17 meses & 15 meses \\
\hline Produção de palavras monossilábicas ou interjeições acompanhadas por gestos & Mais de 17 meses & Mais de 15 meses \\
\hline Produção de palavras onomatopaicas acompanhadas por gestos & Mais de 17 meses & 16 meses \\
\hline Produção de palavras isoladas acompanhadas por gestos & Mais de 17 meses & 17 meses \\
\hline Combinação de duas palavras acompanhada por gestos & Mais de 17 meses & Mais de 17 meses \\
\hline Combinação de mais de duas palavras acompanhada por gestos & Mais de 17 meses & Mais de 17 meses \\
\hline Produção de sílabas com significado não acompanhadas por gestos & Mais de 17 meses & 17 meses \\
\hline Produção de palavras monossilábicas ou interjeições não acompanhadas por gestos & Mais de 17 meses & Mais de 17 meses \\
\hline Produção de palavras onomatopaicas não acompanhadas por gestos & Mais de 17 meses & Mais de 17 meses \\
\hline Produção de palavras isoladas não acompanhadas por gestos & Mais de 17 meses & Mais de 17 meses \\
\hline Combinação de duas palavras não acompanhada por gestos & Mais de 17 meses & Mais de 17 meses \\
\hline Combinação de mais de duas palavras não acompanhada por gestos & Mais de 17 meses & Mais de 17 meses \\
\hline
\end{tabular}

Legenda: GSD = gemelar que apresenta síndrome de Down; GNSD = gemelar que não apresenta síndrome de Down 
Quadro 5. Comparação qualitativa entre os gêmeos em relação ao desenvolvimento cognitivo na avaliação final

\begin{tabular}{|c|c|c|c|}
\hline \multicolumn{4}{|c|}{ Avaliaçâo final - desenvolvimento cognitivo } \\
\hline \multicolumn{2}{|l|}{ GSD } & \multicolumn{2}{|l|}{ GNSD } \\
\hline Realizações da criança & $\begin{array}{c}\text { Fase de } \\
\text { desenvolvimento } \\
\text { cognitivo }\end{array}$ & Realizações da criança & $\begin{array}{c}\text { Fase de } \\
\text { desenvolvimento } \\
\text { cognitivo }\end{array}$ \\
\hline $\begin{array}{l}\text { - procura pelo objeto totalmente escondido, } \\
\text { considerando apenas os deslocamentos } \\
\text { visíveis do objeto; } \\
\text { - imitação de esquemas motores visíveis no } \\
\text { próprio corpo (bater palmas e dar tchau) } \\
\text { - realização de experiências com objetos } \\
\text { novos } \\
\text { - utilização de objetos como meios (condutas } \\
\text { do suporte e do barbante) }\end{array}$ & $\begin{array}{l}5^{a} \text { fase do período } \\
\text { sensório-motor }\end{array}$ & $\begin{array}{l}\text { - procura pelo objeto totalmente escondido, } \\
\text { considerando os deslocamentos visíveis e } \\
\text { invisíveis do objeto; } \\
\text { - imitação de esquemas motores que } \\
\text { designam função aos objetos (empurrar o } \\
\text { carrinho, realizando vibração de lábios, ninar } \\
\text { a boneca) } \\
\text { - realização de experiências com objetos } \\
\text { novos } \\
\text { - utilização de objetos como meios (condutas } \\
\text { do suporte e do barbante) } \\
\text { - uso de esquemas simbólicos simples } \\
\text { (apontar, dar tchau, levar o telefone à } \\
\text { orelha, levar o garfo à boca da boneca, entre } \\
\text { outros) }\end{array}$ & $\begin{array}{l}6^{a} \text { fase do período } \\
\text { sensório-motor }\end{array}$ \\
\hline
\end{tabular}

Legenda: GSD = gemelar que apresenta síndrome de Down ; GNSD = gemelar que não apresenta síndrome de Down

Quadro 6. Comparação qualitativa entre os gêmeos em relação ao desenvolvimento de linguagem expressiva na avaliação final

\begin{tabular}{|c|c|c|c|}
\hline \multicolumn{4}{|c|}{ Avaliaçâo final - desenvolvimento de linguagem expressiva } \\
\hline \multicolumn{2}{|l|}{ GSD } & \multicolumn{2}{|l|}{ GNSD } \\
\hline Produções da criança & $\begin{array}{l}\text { Conjunto de } \\
\text { produções }\end{array}$ & Produções da criança & $\begin{array}{l}\text { Conjunto de } \\
\text { produções }\end{array}$ \\
\hline $\begin{array}{l}\text { - imitação imediata de gestos dêiticos ("cadê", } \\
\text { virando as palmas das mãos para cima) e } \\
\text { representativos (dar tchau, e bater palmas) } \\
\text { - produção de vocalizações isoladas, sem } \\
\text { possibilidade de significação. }\end{array}$ & 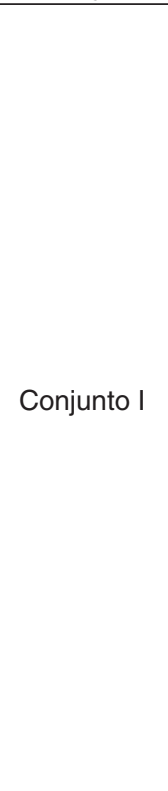 & $\begin{array}{l}\text { - imitação simples de gestos representativos } \\
\text { (levar o telefone à orelha, empurrar o } \\
\text { carrinho); } \\
\text { - imitação diferida de gestos dêiticos (apontar, } \\
\text { chamar com a mão), tanto de forma isolada } \\
\text { quanto acompanhados de vocalizações; } \\
\text { - imitação diferida de gestos representativos } \\
\text { (dar tchau, mandar beijo, bater palmas, levar } \\
\text { o telefone à orelha, levar a colher à própria } \\
\text { boca e à boca da boneca, entre outros), tanto } \\
\text { de forma isolada quanto acompanhados por } \\
\text { vocalizações; } \\
\text { - produção de verbalizações acompanhadas } \\
\text { por gestos ("dá", acompanhada do gesto de } \\
\text { apontar, "auau", apontando para o ursinho de } \\
\text { pelúcia e "mamãe", acompanhada do gesto } \\
\text { de estender os braços para a mãe); } \\
\text { - produção de verbalizações isoladas (sílaba } \\
\text { com significado "bó", ao ver bola). }\end{array}$ & Conjunto IV \\
\hline
\end{tabular}

Legenda: GSD = gemelar que apresenta síndrome de Down; GNSD = gemelar que não apresenta síndrome de Down

cativo deste atraso, provavelmente em função das alterações orgânicas que a acompanham. Esses dados indicam que o atraso no desenvolvimento cognitivo e de linguagem expressiva decorrentes da SD foi agravado pela co-ocorrência da prematuridade e muito baixo peso, evidenciado pelos resultados obtidos para o GSD.

O protocolo utilizado mostrou-se um importante e efetivo instrumento de observação e análise, pois permitiu a obtenção de dados objetivos em relação ao desenvolvimento cognitivo e de linguagem expressiva, bem como a localização e acompanhamento das crianças nesse processo de construção, durante o período sensório-motor.

A partir do PODCLE, foi possível observar que os gêmeos percorreram as mesmas etapas do desenvolvimento cognitivo e de linguagem observadas no desenvolvimento típico, ou seja, seguiram o mesmo caminho proposto na cronologia das 
aquisições como preconizado pela psicogênese, porém em ritmo mais lento.

Nesse sentido, a imitação de esquemas motores, juntamente com a construção do objeto permanente e as relações de tempo, espaço e causalidade, levaram à constituição da representação, evidenciada pelo uso de esquemas simbólicos, gestos dêiticos e representativos, que aos poucos foram sendo acompanhados por vocalizações, sílabas com significados e as primeiras palavras.

Assim, a comunicação gestual continua ativa mesmo quando a linguagem oral começa a emergir, acompanhando as emissões, como manifestação da expansão do repertório de comunicação. Como encontrado por outros autores ${ }^{(8-9)}$, o uso preferencial dos gestos continua por um período de tempo maior nas crianças com SD, dado esse observável no GSD.

A SD e a prematuridade, associada ao muito baixo peso, são, portanto, condições que influenciaram e interferiram de forma negativa no desenvolvimento cognitivo e de linguagem expressiva apresentado pelo par de gêmeos estudado.

\section{COMENTÁRIOS FINAIS}

O objetivo do presente estudo foi descrever o desenvolvimento cognitivo e de linguagem expressiva de uma criança com SD e seu gemelar, ambos nascidos PTMBP, observando a influência da SD e da prematuridade associada ao muito baixo peso no processo de desenvolvimento durante o período sensório-motor.

O estabelecimento de cuidados intensivos em unidades neonatais aliado ao avanço do desenvolvimento tecnológico da medicina obstétrica e perinatal, resultou em aumento das taxas de sobrevida para bebês de alto risco.

Concomitante ao aumento da sobrevivência de recémnascidos cada vez menores e mais imaturos, surge o ques- tionamento quanto à qualidade de vida futura desses bebês, aos aspectos éticos dos limites de viabilidade, ao alto custo da assistência neonatal e aos custos econômicos e sociais dos cuidados pós-alta, no acompanhamento de prematuros com sequelas.

A Fonoaudiologia também tem avançado com marcante participação na atuação terapêutica junto aos indivíduos com SD. A intervenção terapêutica precoce, muitas vezes iniciada no berçário, contribui para a melhoria da qualidade de vida desses indivíduos, tendo o profissional da área se dedicado ao trabalho com a linguagem e a sua relação com os desenvolvimentos neuropsicomotor, cognitivo, emocional e social e sistema estomatognático.

Os resultados deste estudo mostram a influência da prematuridade e muito baixo peso no desenvolvimento cognitivo e de linguagem expressiva, reforçando a idéia de que as crianças nascidas pré-termo e muito baixo peso apresentam risco para alterações ou atrasos no desenvolvimento cognitivo e de linguagem, justificando a elaboração de programas de intervenção fonoaudiológica junto a essas crianças, já durante o primeiro ano de vida.

Crianças com síndrome de Down e histórico de prematuridade e muito baixo peso podem, portanto, apresentar atrasos mais acentuados em relação ao desenvolvimento neuromotor e, consequentemente, em relação ao desenvolvimento cognitivo e de linguagem expressiva.

No entanto, são necessárias mais pesquisas a esse respeito, visto que a maioria dos estudos realizados com crianças com síndrome de Down e crianças pré-termo muito baixo peso, tem mostrado como a linguagem e a cognição se apresentam e não o processo pelo qual a criança chega a tal condição de expressão do conhecimento construído. Além disso, não existem estudos que considerem a síndrome de Down em co-ocorrência com a prematuridade e muito baixo peso.

\begin{abstract}
The aim of this study was to describe expressive language and cognitive development in a child with Down syndrome (DS) and his twin brother, both born preterm very low birth-weight (VLBW), observing the influence of Down syndrome and prematurity combined with very low birth-weight on their development process during the sensorimotor period. Participated in this study a pair of VLBW preterm dizygotic male twins, one of whom presented Down syndrome. The subjects' initial chronological age was seven months and four days and their initial corrected age was four months and 21 days. The twins were born with 29 weeks of gestational age and weighting less than $1500 \mathrm{~g}$. The subjects were followed up during 12 months in 45 -minute fortnight sessions, monthly recorded in video. The Protocol for Expressive Language and Cognitive Development Observation (PELCDO) was used for data gathering and data analysis. Significant differences were observed between the twin brothers concerning expressive language and cognitive development. Although the twin without DS showed better performance, he still presented a relevant delay, considering the references for typically developing children. This finding evidences the influence of prematurity combined with very low birth weight on expressive language and cognitive development. The results found for the other twin suggest that DS led to a significant increase of this delay. DS and prematurity combined with very low birth weight are conditions that negatively interfered on expressive language and cognitive development of the twin pair studied.
\end{abstract}

Keywords: Cognition; Language; Twins, dizygotic; Down syndrome; Infant, premature; Infant, low birth weight; Case reports 


\section{REFERÊNCIAS}

1. Piaget J. Nascimento da inteligência na criança. 3a. ed. Rio de Janeiro: Zahar; 1978. [edição original 1936].

2. Bremner JG. The nature of imitation by infants. Infant Behav Dev. 2002;25(1):65-7.

3. Iverson JM, Goldin-Meadow S. Gesture paves the way for language development. Psychol Sci. 2005;16(5):367-71.

4. Ozçaliskan S, Goldin-Meadow S. Gesture is at the cutting edge of early language development. Cognition. 2005;96(3):B101-13.

5. Fowler A. Language abilities of children with Down syndrome: evidence for a specific syntactic delay. In: Cicchetti D, Beeghly M, editors. Children with Down syndrome: a developmental perspective. US: Cambridge University Press; 1990.

6. Yoder PJ, Warren SF. Early predictors of language in children with and without Down syndrome. Am J Ment Retard. 2004;109(4):285-300.

7. Andrade RV, Limongi SCO. A emergência da comunicação expressiva na criança com síndrome de Down. Pró-Fono. 2007;19(4):387-92.

8. Roberts JE, Price J, Malkin C. Language and communication development in Down syndrome. Ment Retard Dev Disabil Res Rev. 2007;13(1):26-35.

9. Salt A, Redshaw M. Neurodevelopmental follow-up after preterm birth: follow up after two years. Early Hum Dev. 2006;82(3):185-97.
10. Magill-Evans J, Harrison MJ, Van der Zalm J, Holdgrafer G. Cognitive and language development of healthy preterm infants at 10 years of age. Phys Occup Ther Pediatr. 2002;22(1):41-56.

11. Bhutta AT, Cleves MA, Casey PH, Cradock MM, Anand KJ. Cognitive and behavioral outcomes of school-aged children who were born preterm: a meta-analysis. JAMA. 2002;288(6):728-37. Comment in: JAMA. 2002;288(20):2542-3; author reply 2543.

12. Bühler KEB, Flabiano FC, Mendes AE, Limongi SCO. Construção da permanência do objeto em crianças nascidas pré-termo muito baixo peso. Rev CEFAC. 2007;9(3):300-7.

13. Bühler KEB, Flabiano FC, Limongi SCO, Befi-Lopes DM. Protocolo para Observação do Desenvolvimento Cognitivo e de Linguagem Expressiva (PODCLE). Rev Soc Bras Fonoaudiol. 2008;13(1):60-8.

14. Parrat-Dayan S. Processos internos y externos en la construcción de una explicación causal. In: Assis MC, Assis OZM, Ramozzi-Chiarottino Z, organizadores. Piaget: teoria e prática - IV Simpósio Internacional de Epistemologia Genética, XIII Encontro Nacional de Professores do PROEPRE. 1996. p. 28-45.

15. Bühler KECB. Desenvolvimento cognitivo e de linguagem expressiva em bebês pré-termo muito baixo peso em seus estágios iniciais [tese]. São Paulo: Faculdade de Medicina da Universidade de São Paulo; 2008. 